\title{
Error Analysis of Near Field Distribution of Electrostatic Dipole
}

\author{
Yusuke Kusama $^{\mathrm{a},{ }^{*}}$ \\ ${ }^{a}$ NIT Kagawa College, 551 Kouda, Takuma-cho, Mitoyo-city 769-1101, Japan \\ *Corresponding Author: kusama@kagawa-nct.ac.jp
}

\begin{abstract}
Three representation methods for calculating near field distribution of a static electric dipole: 1) exact solution by superposition of single charges, 2) approximate equation using the cosine theorem, 3) multipole expansion including the effect of truncation error are compared. It is confirmed that the radius of the error circle, which is compared with the exact solution, decreases as the number of terms of the multipole expansion increases, but finally it converges to the distance from the origin to the positive or negative point charges. It is found that the electric field inside the circle with point charge as the outer circumference does not coincide with the exact field even if the number of terms of multipole expansion is increased.
\end{abstract}

Keywords: dipole, multipole expansion, education.

\section{Introduction}

The electromagnetic field created by a dipole is essential for learning subjects such as an image method, dielectric polarization, magnetic dipole, magnetization and magnetic poles, small dipole antenna, etc. It can be said that a dipole is a core subject in learning basic electromagnetics. In its formulation, it is well known that an approximation formula using the cosine theorem can be applied when the distance $r$ from the origin to the observation point is sufficiently larger than the distance between both charges $l$, namely in the case of $r>l$. However, there is almost no description or detailed explanations in electromagnetics textbooks about how far the approximation is applicable except for the word "far enough". This is a very interesting matter as a position to teach students about the approximation formula of a dipole field. Therefore, in this study, three formulas including 1) exact field by superimposing single charge, 2) approximate expression using the cosine theorem, 3) multipole expansion

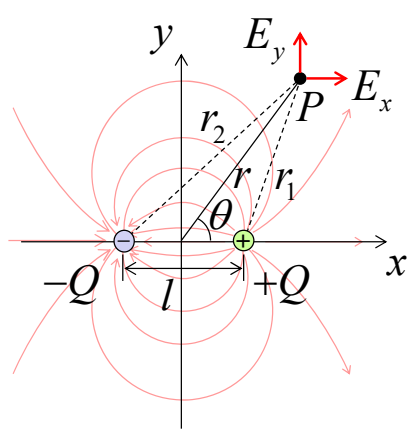

Fig. 1. Calculation model.

representation including the effect of truncation error of expanded terms in Legendre function. Then these application limits were evaluated quantitatively.

\section{Calculation model and formulaes}

We consider a static electric dipole of $\pm Q[\mathrm{C}]$ placed at a distance $l[\mathrm{~m}]$ on the $x$ axis with the origin at the center as shown in Figure 1. The distance from the origin to the observation point is $r$ and the distances from each point charge to the observation point is $r_{1}$ and $r_{2}$, respectively.

\subsection{Exact solution}

The exact expression not including any approximation is represented by Eq. (1) and (2) by superposition of electric potentials created by independent charges ${ }^{[1]}$.

$$
\begin{aligned}
& V=\frac{Q}{4 \pi \varepsilon_{0}}\left(\frac{1}{r_{1}}-\frac{1}{r_{2}}\right), \\
& E_{x}=-\frac{\partial V}{\partial x}=\frac{Q}{4 \pi \varepsilon_{0}}\left(\frac{x-l / 2}{r_{1}^{3}}-\frac{x+l / 2}{r_{2}^{3}}\right), \\
& E_{y}=-\frac{\partial V}{\partial y}=\frac{Q}{4 \pi \varepsilon_{0}}\left(\frac{y}{r_{1}^{3}}-\frac{y}{r_{2}^{3}}\right)
\end{aligned}
$$


where $\quad r_{1}=\sqrt{(x-l / 2)^{2}+y^{2}}, \quad r_{2}=\sqrt{(x+l / 2)^{2}+y^{2}}$

\section{$2.2 \quad$ Approximate expression}

Approximate expressions of Eq. (1) and (2) in the case of $r>l$ are given by Eq. (3) and (4), respectively by using the cosine theorem to express the distance from the point charge to the observation point $r_{1}$ and $r_{2}$ by the distance from the origin $r$ and the angle $\theta$. This approximate expression can be seen in almost all electromagnetics textbooks.

$$
\begin{aligned}
& V=\frac{Q}{4 \pi \varepsilon_{0}} \frac{l}{r^{2}} \cos \theta, \\
& E_{r}=\frac{Q l}{2 \pi \varepsilon_{0}} \frac{\cos \theta}{r^{3}}, \quad E_{\theta}=\frac{Q l}{4 \pi \varepsilon_{0}} \frac{\sin \theta}{r^{3}}, \quad E_{\varphi}=0,
\end{aligned}
$$

where $\quad r=\sqrt{x^{2}+y^{2}}$,

$$
\cos \theta=\frac{x}{\sqrt{x^{2}+y^{2}}}, \quad \sin \theta=\frac{y}{\sqrt{x^{2}+y^{2}}}
$$

In order to unify the coordinate system, Eq. (4) expressed in polar coordinates is transformed into $x, y$ coordinates, it becomes following Eq. (5).

$$
\begin{aligned}
& E_{x}=E_{r} \cos \theta-E_{\theta} \sin \theta, \\
& E_{y}=E_{r} \sin \theta+E_{\theta} \cos \theta
\end{aligned}
$$

\subsection{Multipole expansion}

Multipole expansion around the origin is expressed by Eq. (6) and (7) using Legendre function ${ }^{[2][3]}$.

$$
\begin{aligned}
& V=\frac{Q}{4 \pi \varepsilon_{0} r}\left[\sum_{n=0}^{\infty}\left\{1-(-1)^{n}\right\} P_{n}(\cos \theta)\left(\frac{l / 2}{r}\right)^{n}\right], \\
& E_{x}=-\frac{\partial V}{\partial x}, \quad E_{y}=-\frac{\partial V}{\partial y}
\end{aligned}
$$

where $r=\sqrt{x^{2}+y^{2}}, \quad \cos \theta=\frac{x}{\sqrt{x^{2}+y^{2}}}$

By considering the magnetic potential $V_{m}$ with respect to the electric potential $V$, the same formulation can be applied to a magnetic dipole. Specifically, by substituting the electric charge $Q \Leftrightarrow$ magnetic charge $Q_{m}$ and the reciprocal of dielectric constant $1 / \varepsilon_{0} \Leftrightarrow$ permeability $\mu_{0}$, the magnetic flux density $\boldsymbol{B}$ can be derived corresponding to the electric field $\boldsymbol{E}$. And the magnetic field $\boldsymbol{H}$ can be derived corresponding to the electric flux density $\boldsymbol{D}$.

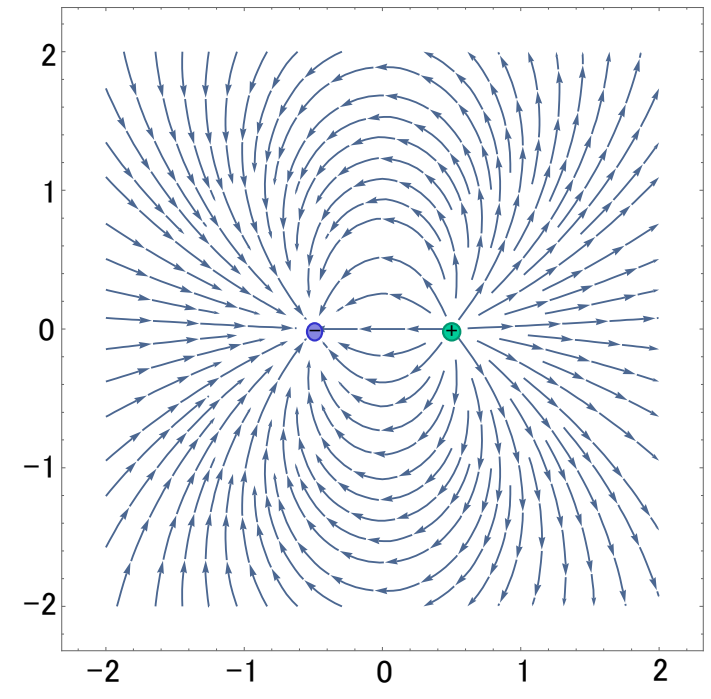

(a) Exact field.

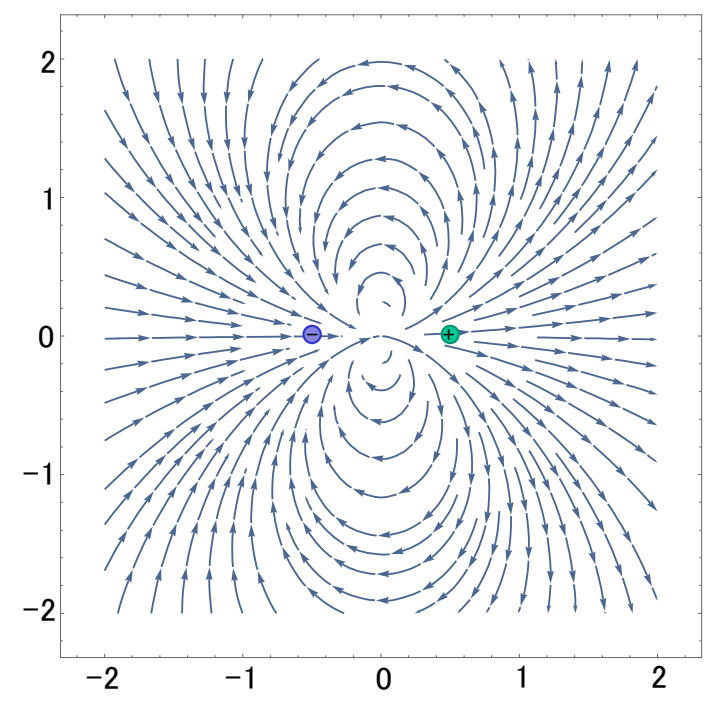

(b) Approximate expression.

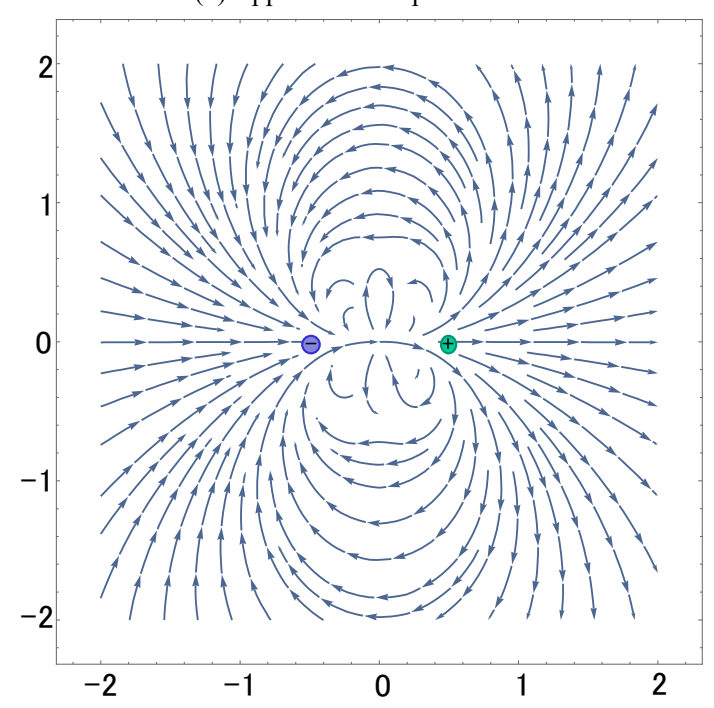

(c) Multipole expansion $(n=3)$.

Fig. 2. Image of electric lines of force near a dipole. Exact field (upper), approximate expression (center), multipole expansion $n=3$ (lower) 
For the sake of simplicity, in this study, as a calculation model condition in the Fig. 1, a negative point charge $-1 \mathrm{C}$ was positioned at the coordinate $(x, y)=(-1 / 2,0)$, and a positive point charge $+1 \mathrm{C}$ is arranged at $(x, y)=(1 / 2,0)$. That is, we consider a model in which the electric dipole moment with $p=Q l=1$ oriented in the positive direction on the $x$ axis is in the origin. In the partial differential calculation of the Eq. (7) including the Legendre polynomial expressed by infinite series, in order to avoid complication of the formulation due to the difference in the number of expansion terms, the differential calculation function of mathematical software Mathematica v11.1 was used.

\section{Comparison of electric field patterns}

\subsection{Comparison of three methods}

Figure 2 shows the image of electric lines of force near the dipole. Exact solution, approximate expression, and multipole expansion in the case of $n=3$ are shown from top to bottom. The approximate expression completely matches the case $n=1$ in the multipole expansion in Eq. (7). That is, the Eq. (7) is a generalized expression of the approximation Eq. (4). The drawing range is set to $[-2,+2]$ for both $x$ and $y$. The StreamPlot function of Mathematica v11.1was used for drawing electric lines of force image. In any case, space outside the circle with the radius $r=1 / 2$, where the positive and negative point charges are as the center around the origin, a typical figure-of-eight electric field pattern can be observed. However, in the space inside the circle with the radius $r=1 / 2$, it can be observed the difference that the direction of the vector is reversed on the $x$ axis and the strong spin occurs in the vicinity thereof, as compared with the exact solution for both the approximate expression and the multipole expansion. The strength of the spin becomes stronger as the number of multipole expansion $n$ increases.

In order to observe this difference in more detail, contour lines for each of $E_{x}$ and $E_{y}$ components are shown in Figure 3. The drawing range is set to $[-2,+2]$ for both $x$ and $y$. The left column shows $E_{x}$ and the right column shows $E_{y}$ and three rows show the exact solution, approximate expression $n=1$, multipole expansion $n=3$ in order from the top to bottom as in Fig. 1. The drawing range of the contour line is $\pm 15 \times 10^{9} \mathrm{~V} / \mathrm{m}$, and it is drawn every $5 \times 10^{9} \mathrm{~V} / \mathrm{m}$. For example, comparing exact solutions and approximate expressions, $E_{y}$ is a relatively similar pattern, whereas $E_{x}$ in the approximate expression shows

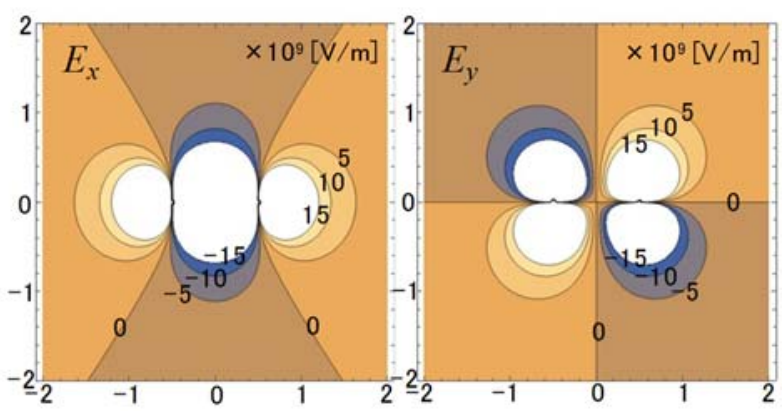

(a) Exact solution

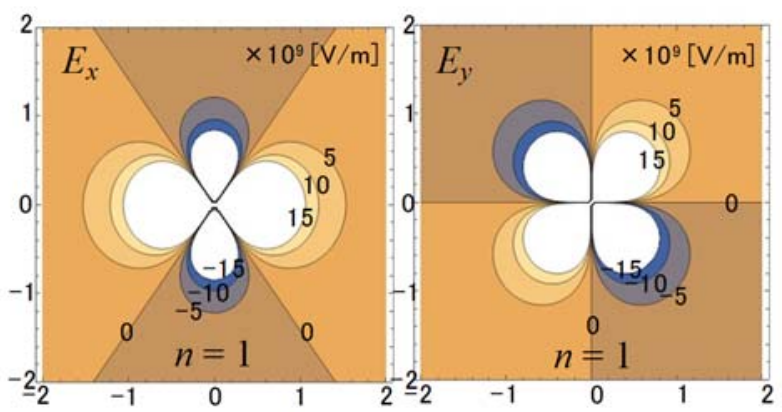

(b) Approximate expression.

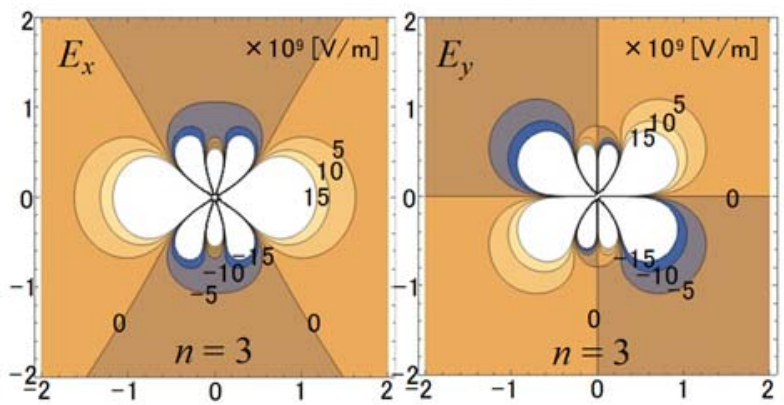

(c) Multipole expansion $n=3$.

Fig. 3. Electric field distribution near dipole. The left column shows $E_{x}$, the right column shows $E_{y}$, from the top shows exact solution, approximate expression, multipole expansion $n=3$.

that the pattern clearly differs, such as the occurrence of a zero at the origin. In addition, when exact solution and multipole expansion $n=3$ are compared, it seems that both $E_{x}$ and $E_{y}$ coincide well when going away from the origin, but the influence of the higher order function of Legendre polynomial appears conspicuously in the vicinity of the origin, it is obvious that the pattern is clearly different. In this way, it can be seen that neither the approximate expression nor the multipole expansion can sufficiently express the exact dipole field in the vicinity of the origin, and in particular the effect of the vibration given to the vicinity of the origin by the influence of the higher order 


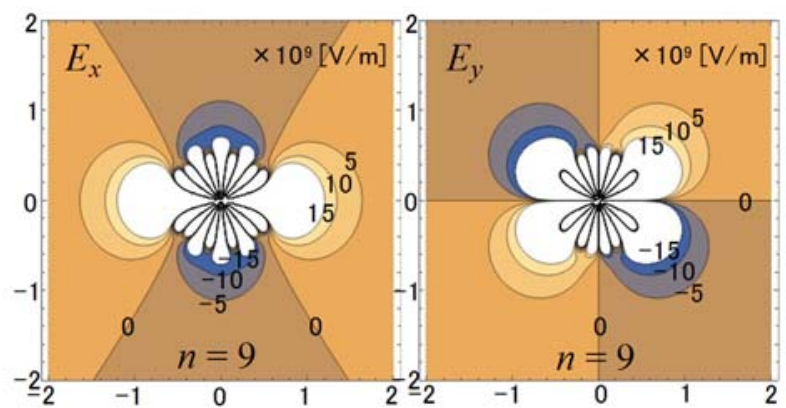

(a) Case of $n=9$.

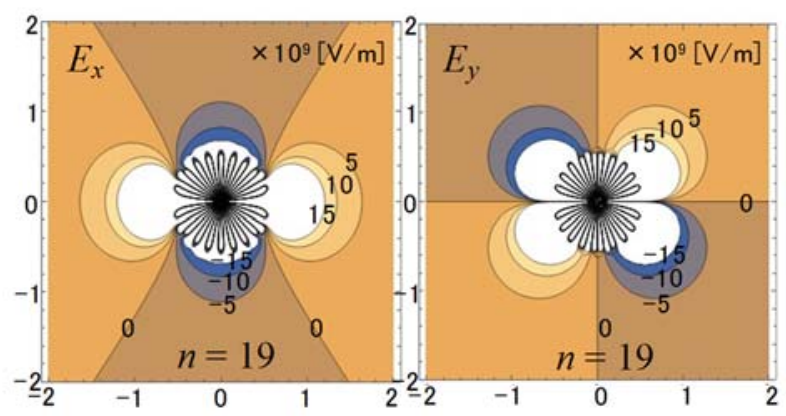

(a) Case of $n=19$.

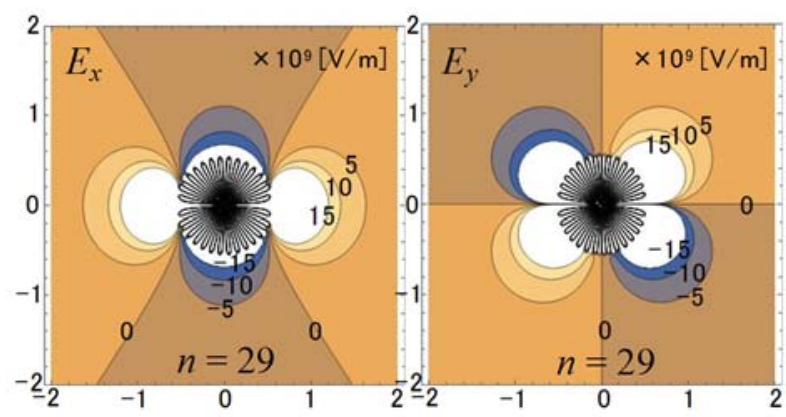

(b) Case of $n=29$.

Fig. 4. Examination of the number of truncation terms of multipole deployment. The left column shows $E_{x}$, the right column shows $E_{y}$, and the case of $n=9,19,29$ from the top is shown.

pattern of the Legendre function is remarkable.

\subsection{Examination of the number of truncation error of multipole expansion}

In the previous section, we compared the exact solution up to multipole expansion $n=3$. When using a function expressed by a series as in the Legendre function, generally there is a property that it matches an exact solution as the number of truncated terms $n$ is increased. Therefore, we examined the case where the number of truncated terms of multipole expansion was increased. Figure 4 shows contour lines for each of the $E_{x}$ and $E_{y}$ components. The case of $n=$

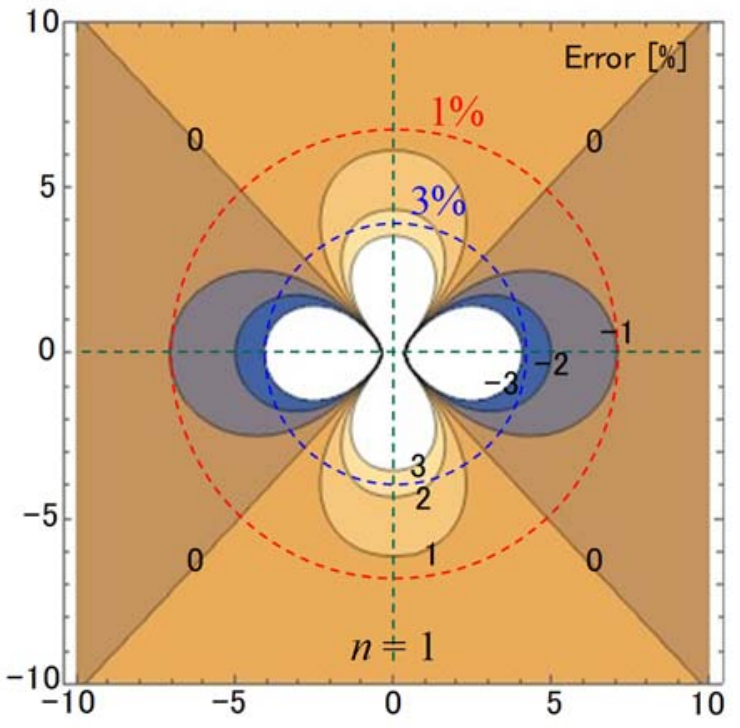

(a) Case of $n=1$.

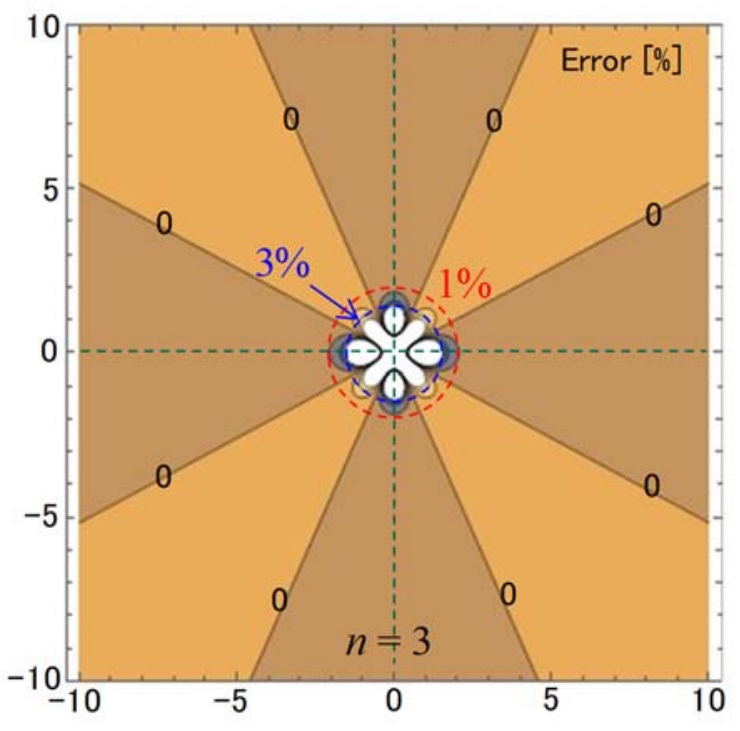

(b) Case of $n=3$.

Fig. 5. Error distribution near the dipole. Approximate equation $n=1$ (upper) Multipole expansion $n=3$ (lower) is shown.

9, 19, and 29 from the top are shown. The drawing range and the interval between the contour lines are the same as in Fig. 3. The reason why the numbers of multipole expansion are all unified to odd numbers is that the even term of the potential disappears automatically from Eq. (6). From the figure, it is possible to remarkably observe the difference of the pattern of $\pm 5 \times 10^{9} \mathrm{~V} / \mathrm{m}$ on the outermost side among the contour lines drawn at $n=9$. However, at $n=19$, this difference disappears, and the vicinity of the origin due to the higher order pattern also tends to fall within the circular range of approximately $r=1 / 2$. At $n=29$, the innermost \pm $15 \times 10^{9} \mathrm{~V} / \mathrm{m}$ pattern of the contours drawn is closer to the 
exact solution, but the circular range that oscillates higher in the vicinity of the origin hardly changes.

\subsection{Error analysis}

As shown in Fig. 4, when the number $n$ of is increased, the pattern outside the circle with the radius $r=1 / 2$ where the positive and negative point charges are as the center around the origin approaches the exact solution. To evaluate this variation quantitatively, the error evaluation is defined by the following Eq. (8).

$$
\text { Error }=\frac{\left|E_{n}\right|-\left|E_{\text {True }}\right|}{\left|E_{\text {True }}\right|} \times 100[\%]
$$

Here, $E_{\text {True }}$ is the square root of sum of squares of the exact solution Eq. (2), $E_{\mathrm{n}}$ is the square root of sum of squares of the approximation Eq. (4) or multipole expansion Eq. (7). The error distributions of the approximate expression $n=1$ and the multipole expansion $n=3$ are shown in Figure 5 . Both $x$ and $y$ are drawn in the range of $[-10,+10]$ which is the same as in Fig. 2. The drawing range of the contour line is $\pm 3 \%$, and is drawn every $\pm 3 \%, \pm 2 \%$, and $\pm 1 \%$. In the case of the approximate expression $n=1$, the error on the $x$ axis on which the dipole axis is placed is slightly larger than that on the $y$ axis, and its value becomes negative (a value smaller than the exact solution). The radius $r$ at which this value is $-1 \%$ is indicated as $1 \%$ error circle, and the radius $r$ at $-3 \%$ is written as 3\% error circle in the figure. For example, the radius of the $1 \%$ error circle is about $r=7$, and the radius of the $3 \%$ error circle is about $r=4$. When normalized by the distance $l$ between the positive and negative point charges, it coincides with an exact solution

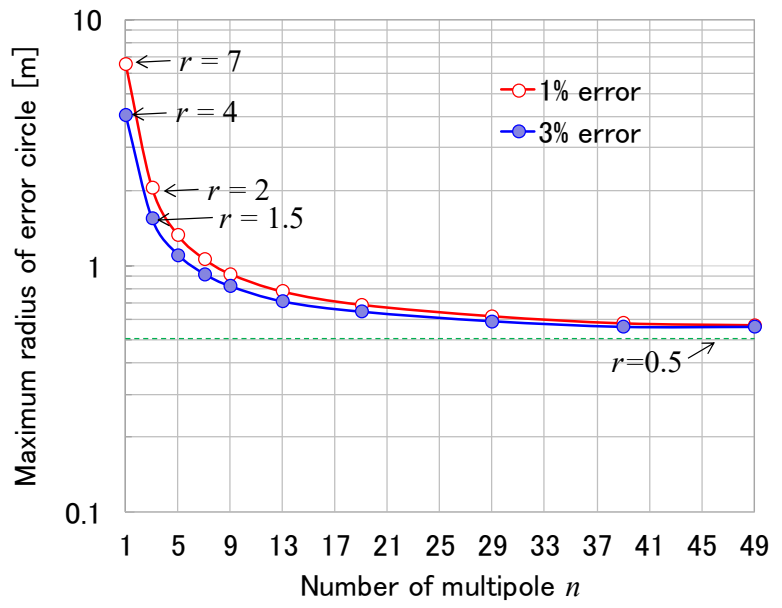

Fig. 6. Examination of the number of truncation terms of multipole deployment. with an error of $1 \%$ if it is separated by $r=7 l$. And if it is separated by $r=4 l$, it coincides with an exact solution with an error of $3 \%$. Also in the case of $n=3$, the radius of the $1 \%$ error circle is $r=2 l$ and the radius of the $3 \%$ error circle is $r=1.5 l$. Figure 6 shows how the error radius varies with increasing expansion number $n$. Although the error radius sharply decreases up to $n=9$, it can be seen that there is no significant improvement even if $n$ is increased after that but finally it gradually approaches the distance $r=0.5 l$ which is the distance from the origin to the point charge.

\section{Conclusions}

For the electrostatic field distribution in the vicinity of the dipole, three representation methods including 1) exact solution by superposition of single charge, 2) approximate equation, 3) multipole expansion including the effect of truncation error were compared. In the comparison of electric field distribution contours, it was found that the multipole expansion agrees well with the exact solution in the space outside excluded the point charges when the expansion term number is increased. However, in the space inside the point charge, both the approximate expression and the multipole expansion have significantly different distributions as compared with the exact solution, and even if the number of expanded terms of the multipole expansion is increased, the influence of the vibration by the higher order pattern becomes conspicuous. So it is not possible to accurately represent the exact dipole field. From the comparison of the error distribution contours, it was confirmed that the radius of the error circle decreases as the number of expansion terms of the multipole expansion increases, but finally it converges to the distance from the origin to the positive and negative point charges.

\section{References}

(1) Saburo Aadachi, and Shigeo Onuki: "Electromagnetics $2^{\text {nd }}$ ed.", Morikita Publishing Co., pp. 33-34, 2014

(2) Yasufumi Kawamura, Kazuo Umemura, Daiki Katoh, , Kazuo Kitahara, Hideaki Sakata, Katuhiko Suzuki, Kiyoshi Torizuka, and Yoshikazu Honma : "Easy-to-understand science and engineering electromagnetism", Koudansha Publishing Co., pp. 40-43, 2012

(3) Hideo Nitta: "Physics and Special Functions Introductory seminar -", Kyoritsu Publishing Co., pp.37-40, 1997 